\title{
Properties and applications of Magnetorheological fluids
}

\author{
A. Spaggiari \\ Dept. of Engineering Sciences and Methods, University of Modena and Reggio Emilia, Italy \\ andrea.spaggiari@unimore.it
}

ABSTRACT. This brief introduction describes the mechanical, rheological and magnetic properties of the magnetorheological (MR) fluids for feasible engineering applications. The typical modes of exploiting this technology are shown and discussed. An increasing number of industrial applications illustrate how the MR fluids peculiar properties may be used to provide optimal performance in semi active damping and dissipative devices.

KEYWORDS. Magnetorheological fluids; Pressure; Shear mode; Interaction; Experimental.

\section{Properties of MAgnetorheological FLUIDS}

$\mathrm{M}$ agnetorheological (MR) fluids are smart and controllable materials, even though at the first glance they do not look so impressive. They are a non colloidal mixture of ferromagnetic particles randomly dispersed in oil or water (Fig. 1a), plus some surfactants useful to avoid the settling of the suspended particles. The overall aspect is like a greasy quite heavy mud, since MR fluids density is more than three times the density of water. This material becomes suddenly smart and interesting as soon as a magnetic field passes through it. The ferromagnetic particles feel the induction field and acquire a magnetic bipole, then they move and redesign their arrangement start to flow and to form chains and linear structures (Fig. 1b). These microscopic chains have a the macroscopic effect to change the apparent viscosity of the fluid. The size of the particles is around 10 micron for the so called MR fluids (Fig. 2a) mainly produced by [1], while the nanosized particles produces a similar substance called ferrofluid (Fig. 2b), produced by [2]. While the applications of MR fluids are relevant for engineers and can be used in many damping devices, the ferrofluids are mainly a fancy stuff to play with for artist and kids. The reason for this distinction is simple: there is a huge difference in the yield shear stress of the MR and ferrofluids, which affects the maximum force the fluid can provide.

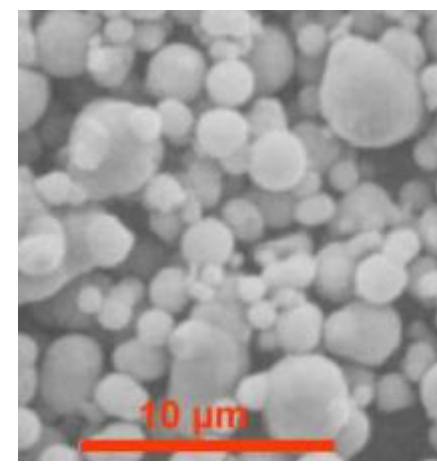

(a)

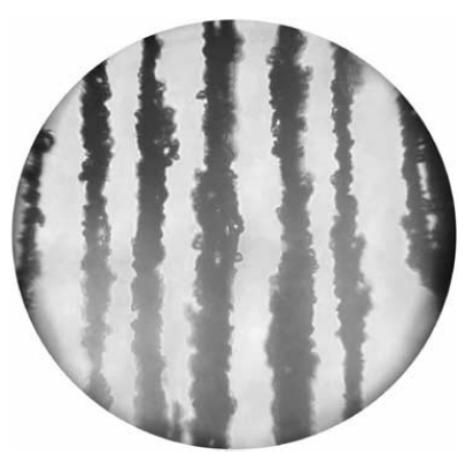

(b)

Figure 1: Microfotography of a MR fluid with no magnetic field, where particles are randomly dispersed (a). Microphotography MR fluid with an applied magnetic field with parallel chains of carbonyl iron (b). 


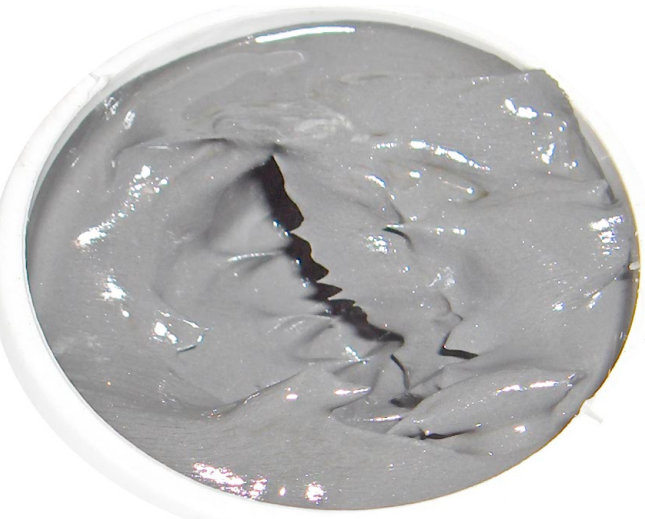

(a)

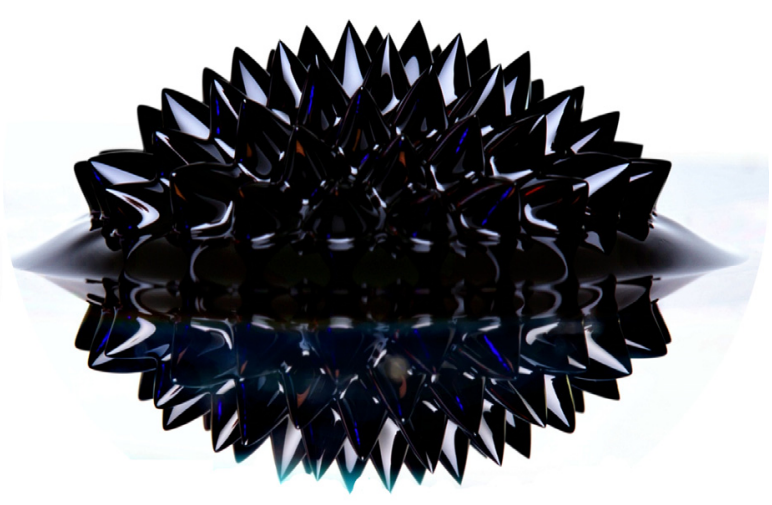

(b)

Figure 2: Magneto rheological fluid (a) from Lord [1] and ferrofluid (b), produced by [2].

The yield shear stress is the main figure of merit of a MR fluid and derives from the non Newtonian behaviour of these fluids. The MR fluid behaves following a so called Bingham law, which means that it exhibits a non zero shear stress value for a zero shear rate [3], behaving more like a solid than like a liquid, as shown in Fig. 3a. The value of the shear stress at no shear rate is called yield stress of the MR fluid and is controlled by the applied magnetic field as shown in Fig. $3 \mathrm{~b}$. The larger the field, the higher the yield stress. The higher the yield stress the higher the force the material can withstand without flowing. Bearing a load is possible only because MR fluids can modify their aggregations states changing from a viscous free-flow liquid to a quasi solid state.

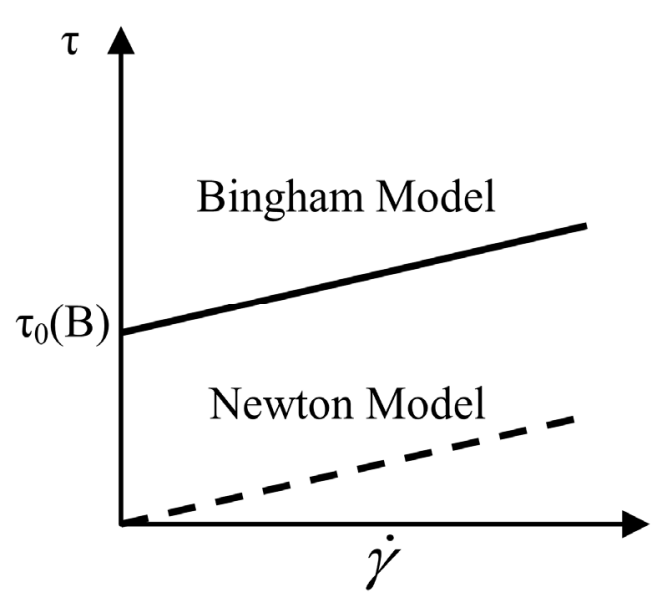

(a)

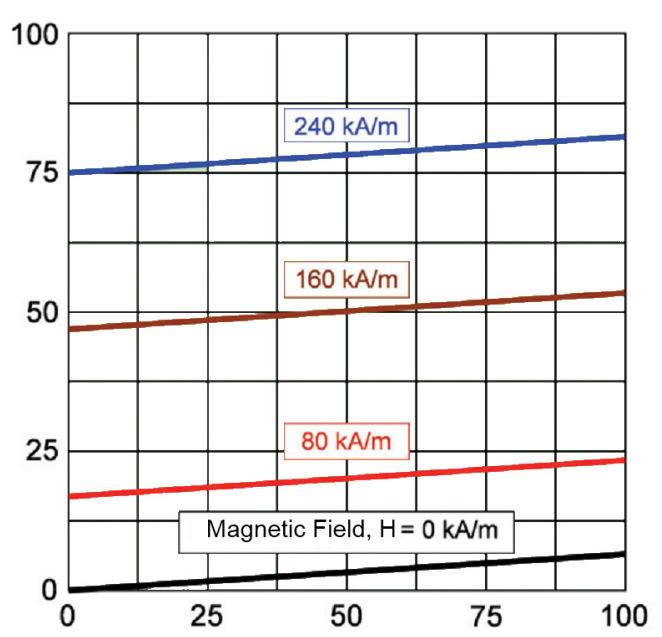

(b)

Figure 3: Bingham model of MR fluid (a) and effect of the magnetic field on the yield stress (b).

In order to exploit MR fluids properties there are three main way envisioned in current engineering applications [4]:

a) Flow mode, shown in Fig. 4a;

b) Shear mode, shown in Fig. 4b;

c) Squeeze mode, shown in Fig. 4c.

Flow mode, also called valve mode, exploits the fluid between two fixed walls, the magnetic field is normal to the flow directions and is typical for linear damper applications. Shear mode is mainly used in rotary application such as brakes and clutches and the fluid is constrained between two walls which are in relative motion with the magnetic field normal to the wall direction. Squeeze mode is used mainly for bearing applications, is able to provide high forces and low displacements having the magnetic field normal to walls directions. In all the above mentioned cases the working principle is the same: the applied magnetic field regulates the yield stress of the fluid and changes its apparent viscosity. So the amount of 
dissipated energy of the system is simply controllable by acting on the coil current and the system can provide semi-active behaviour.

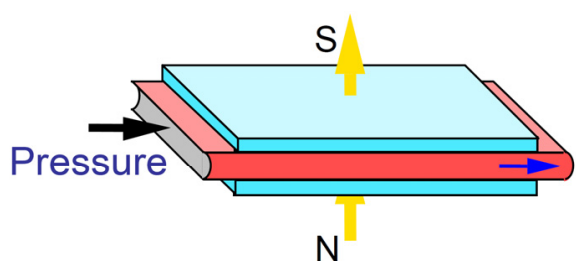

Magnetic field $\boldsymbol{B}$

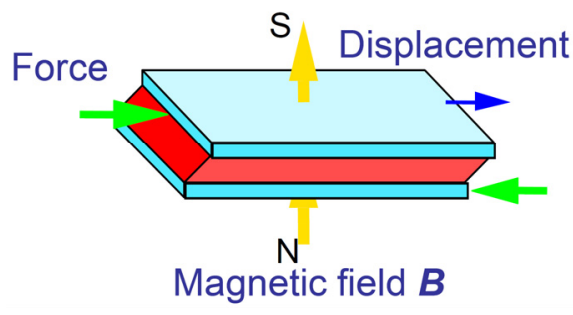

(b)

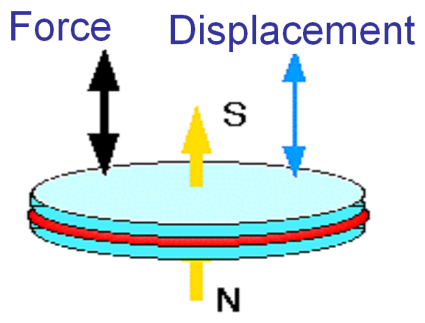

Magnetic field $B$

(c)

(a)

Figure 4: Typical using modes for MR fluids, flow (a), shear (b) and squeeze (c) mode.

\section{APPLICATIONS OF MAGNETORHEOLOGICAL FLUIDS}

7 he sudden change in the MR behaviour (few milliseconds) due to the magnetic field application, makes this material attractive for damping and dissipative devices. The MR fluids can be used to build integral, silent, quick mechanical systems enhanced by means of electronic controls.

The MR applications in the field of are semi-active control devices which offer the flexibility and versatility of the active systems and the reliability of the passive ones. There are two main ways to exploit the MR fluids in engineering applications.
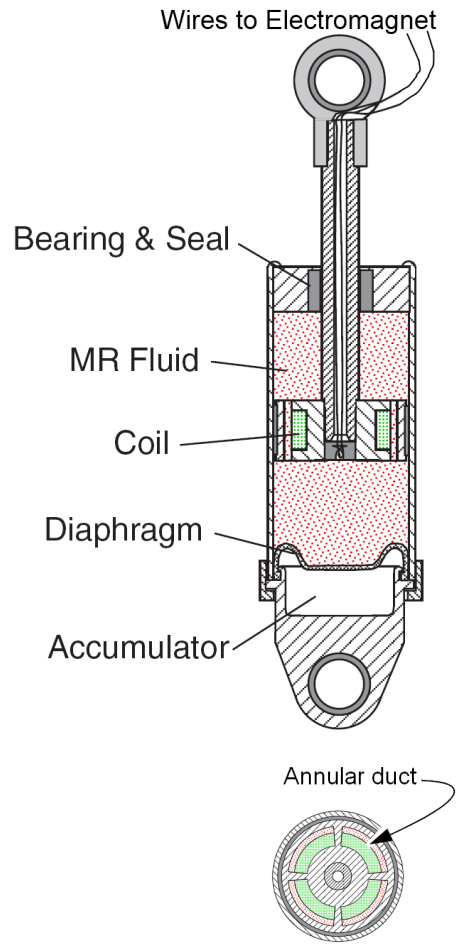

Damper cross section

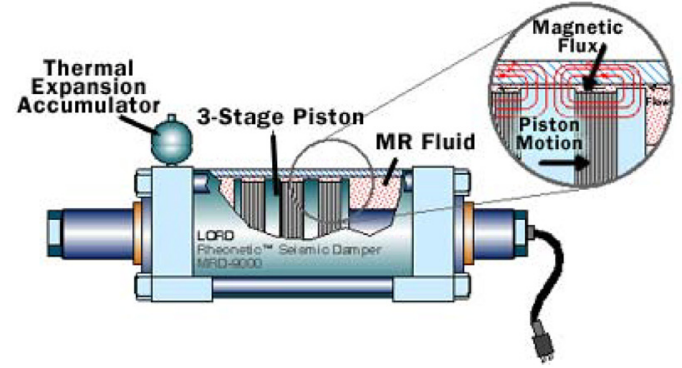

(b)

(a)

Figure 5: Schematic of single ended MR damper and double ended MR damper, both produced by Lord [1]. 


\section{Linear MR devices}

One of the most interesting engineering applications of MR fluid is the construction of smart and controllable MR linear dampers. The main asset of a MR based damper is the controllability of the system, which can be adjusted in order to provide the desired level of damping by simply changing the supply current. The main idea is to obtain the desired level of damping by varying the magnetic induction in an orifice between two separated MR fluid chambers. The orifice acts like a magnetic valve for the fluid, regulated by the current and thus exploits the MR fluid in flow mode. Two many architectures are envisioned for this purpose: the single ended damper (Fig. 5a) and the double ended damper (Fig. 5b). The single ended damper has only one reservoir for the MR fluid which is transferred through an orifice from on chamber to another. Since the rod volume is just on one side the system accounts for the change in volume that results from piston rod movement. In order to accommodate this change in reservoir volume, an accumulator is usually used. The accumulator provides a barrier between the MR fluid and a compressed gas (usually nitrogen) that is used to accommodate the necessary volume changes. Moreover the accumulator pressure can be used to enhance the performance of the MR system, as shown in [5]. The double-ended MR damper has piston rods of the same diameter that protrude through both ends of the damper. In this case there is no change in volume as the piston rod moves, the doubleended damper does not require an accumulator or other similar device. The applications of the single ended damper are mainly in the vibration suppression of mechanical components like seat suspension, car suspensions, and industrial vibration suppression, while the double ended damper is mainly used for bicycle applications, gun recoil applications, and for stabilizing buildings and bridges during earthquakes. The output forces of such a devices can range from quite low forces (hundreds of Newtons) in case of light suspension system up to 20 tons in case of civil applications, in which they must compensate the incredibly large forces cause by the shaking of entire buildings.

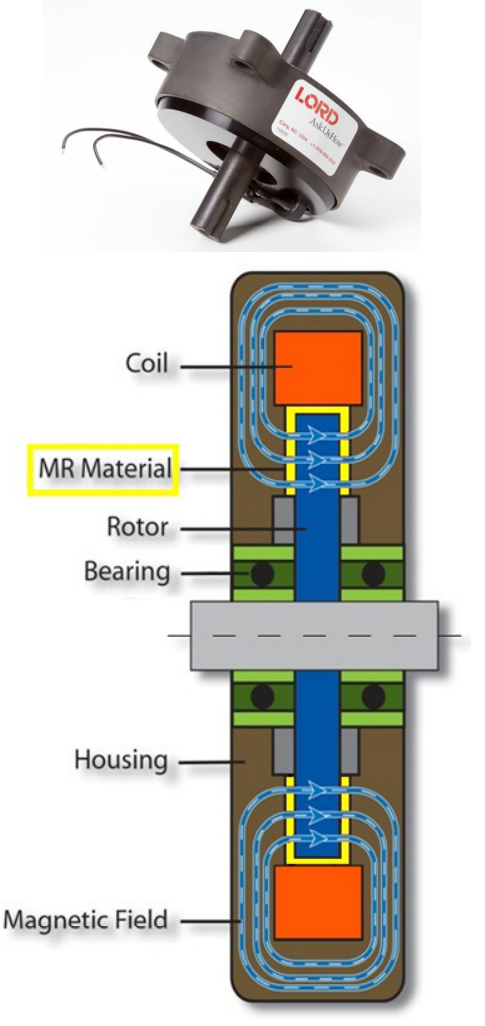

(a)

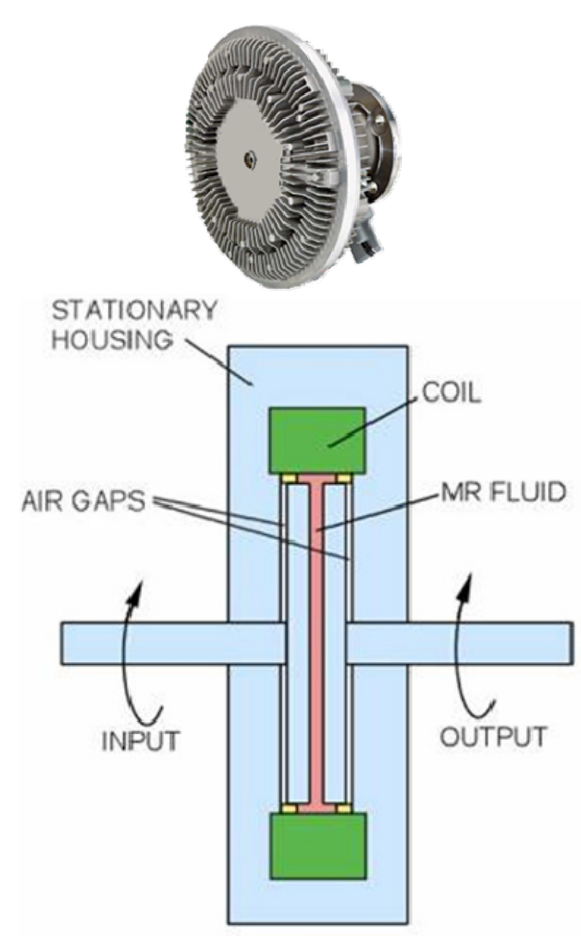

(b)

Figure 6: Picture and schematic of MR based brake (a) and clutch, both produced by Lord [1].

\section{Rotary MR devices}

The other main application exploits the MR fluid in shear mode to realize a sort of hydraulic brakes and clutches with MR fluids. The aim is to obtain a precise control of the braking torque (in case of brakes) or transmitted torque (in case of clutches) with no moving parts by simply varying the current in the coils. The typical architecture for a MR based brake is depicted in Fig. 6a, where the basic idea is clearly depicted. The magnetic flux path passes through the chassis and the 
rotating disk and the fluid is sheared between these elements. The braking force depends on the yield stress of the fluid making the system controllable. The MR based clutch is ideally described by Fig. 6b, the fluid is between the input disk and the output disk and the amount of transmitted torque is proportional to the yield stress of the fluid. No moving part are used to change the transmitted torque and the torque value can be smoothly controlled through the coil current. Even though multidisc applications can be used to increase the output torque, the typical application of rotary MR fluid devices is in the high precision and low power range. For example the Rheoknee, developed by Ossur [6] is the first prosthetic knee which allow the amputee to have a normal leg motion even in case of stairs. The MR knee exhibits a very low torque when is not active enabling the leg to move forward freely and in a few millisecond the MR is able to carry all the human weight to complete the step. The overall performance is outstanding and shows how the smartness of MR fluid can improve human condition.

\section{CONCLUSION}

7 he world of dissipative and damping device is full of potential MR fluid applications, especially when it is desirable to control motion. Traditional viscosity based system with moving parts can be easily replaced by a MR fluid with changing viscosity as function of current, obtaining an improvement in functionality and a costs reduction. The MR fluid main features are: fast response, simple interface between electrical power input and the mechanical power output, controllability and integration in complex system. Nowadays MR fluid are a reliable technology for many engineering applications. Flow mode (used in dampers) and shear mode (used in brakes and clutches) have been studied thoroughly and several products are already present in the market.

\section{REFERENCES}

[1] Lord Corp. http://www.lord.com/products-and-solutions/magneto-rheological- $\% 28 \mathrm{mr} \% 29 . \mathrm{xml}$

[2] Apexmagnets LLC http://apexmagnets.com/index.php?main_page=index\&cPath=7

[3] E. Dragoni, Progettare, 332 (2009).

[4] M. R. Jolly, J. W. Bender, J. D. Carlson, J. Intell. Mater. Syst. Struct., 10 (1999) 5.

[5] A. Spaggiari, E. Dragoni, ASME J. Fluids Eng., 134 (2012) 091103.

[6] Ossur Company http://www.ossur.com/?PageID=15766 